\title{
Standard of disocclusion in complete dentures supported by implants without free distal ends: analysis by the finite elements method
}

\author{
Gustavo Diniz GRECO1 ${ }^{1}$, Estevam Barbosa de LAS CASAS ${ }^{2}$, Tulimar P. Machado CORNACCHIA ${ }^{3}$, Cláudia Silami de \\ MAGALHÃES $^{3}$, Allyson Nogueira MOREIRA ${ }^{3}$
}

\author{
1- PhD student, Dental School, Federal University of Minas Gerais, Belo Horizonte, MG, Brazil. \\ 2- PhD, Professor, Structural Engineering Department, School of Engineering, Federal University of Minas Gerais, Belo Horizonte, MG, Brazil. \\ 3- DDS, MS, PhD, Associate Professor, Department of Restorative Dentistry, School of Dentistry, Federal University of Minas Gerais, Belo Horizonte, MG, Brazil.
}

Corresponding address: Gustavo Diniz Greco - Rua Pedra Bonita, 924 - Barroca - 30360-390 - Belo Horizonte, MG - Brazil - Phone/fax: 55-31-3334-3673

- Cel: 31-84551945 - e-mail: gustavodgreco@yahoo.com.br

Received: November 16, 2009 - Modification: April 29, 2010 - Accepted: October 26, 2010

\section{ABSTRACT}

\begin{abstract}
bjective: The occlusal patterns are key requirements for the clinical success of oral rehabilitation supported by implants. This study compared the stresses generated by the disocclusion in the canine guide occlusion (CGO) and bilateral balanced occlusion (BBO) on the implants and metallic infrastructure of a complete Brånemark protocol-type denture modified with the inclusion of one posterior short implant on each side. Material and Methods: A three-dimensional model simulated a mandible with seven titanium implants as pillars, five of them installed between the mental foramen and the two posterior implants, located at the midpoint of the occlusal surface of the first molar. A load of $15 \mathrm{~N}$ with an angle of $45^{\circ}$ was applied to a tooth or distributed across three teeth to simulate the CGO or BBO, respectively. The commercial program ABAQUS ${ }^{\circledR}$ was used for the model development, before and after the processing of the data. The results were based on a linear static analysis and were used to compare the magnitude of the equivalent stress for each of the simulations. Results: The results showed that the disocclusion in CGO generated higher stresses concentrated on the working side in the region of the short implant. In $\mathrm{BBO}$, the stresses were less intense and more evenly distributed on the prosthesis. The maximum stress found in the simulation of the disocclusion in CGO was two times higher than that found in the simulation of the BBO. The point of maximum stress was located in the neck of the short implant on the working side. Conclusions: Under the conditions of this study, it was concluded that the BBO pattern was more suitable than CGO for the lower complete denture supported by implants without free distal ends.
\end{abstract}

Key words: Dental occlusion. Dental implants. Biomechanics.

\section{INTRODUCTION}

A complete mandibular fixed denture with five or six implants as pillars and free bilateral distal ends was proposed by Brånemark² (1983). Since then, researchers have tried to identify and demonstrate the most appropriate occlusal factors capable of providing a smooth and efficient disocclusion and also to understand their relationships with the stomatognathic system ${ }^{4,9,16,18,25}$. During this period, the associations between the occlusal factors and the mastication muscles, chewing efficiency, bruxism, temporomandibular joint and adjacent tissues have been investigated. The canine guide is frequently used as a standard in the physiological movements of the natural dentition ${ }^{21,24}$. Otherwise, few consistent conclusions and minimal scientific support concerning the occlusal patterns applied to the complete denture supported by implants are available.

The occlusal pattern can be considered a critical factor for the longevity of the components of stomatognathic system, including integrated implants. In the natural dentition, the periodontal ligament acts as a damping system that improves 
the absorption of occlusal stresses. Because there is no periodontal ligament at the implant-bone interface, stress distribution in the prosthesis, its components, the implants and the implant-bone interface is simpler than in the natural dentition. If the occlusal forces exceed the capacity of the system, oral rehabilitation fails due to overload and a poor load distribution ${ }^{4,18,25}$.

The Brånemark protocol was developed to be the antagonist of conventional complete denture ${ }^{2}$. The technological evolution applied to surgical techniques and to the design and surface treatment of implants has allowed the use of this type of prosthesis with antagonists supported by implants, teeth or joint prostheses. There are clinical reports that the stress distribution generated in the functional loads may surpass the implant's strength and cause, with some frequency, fracture of prostheses with free distal ends ${ }^{14,20,25}$.

Using finite element methods (FEM), Greco, et al. ${ }^{8}$ (2009) investigated the stresses generated by different patterns of disocclusion - canine guide occlusion (CGO) and bilateral balanced occlusion (BBO) - in a three-dimensional (3D) model of a mandibular complete denture supported by implants with free distal ends. The results showed the following: (I) the pattern of disocclusion in CGO led to increased stresses in the implant in the region of the canine on the working side, and (II) in the BBO, stresses were high throughout the infrastructure. It was concluded that the pattern of disocclusion in CGO was ideal for the mandibular complete denture supported by implants of the Brånemark protocol type.

Currently, unlike at the time when the traditional protocol was developed, the option of including bilateral short implants is available, eliminating the free distal ends. When a bilateral free distal end is eliminated, this prosthesis approaches the concept of the fixed rehabilitation setting and deviates from the removable rehabilitation concept. However, the question remains as to the pattern of disocclusion to use. Disocclusion in CGO follows the philosophy applied to fixed prostheses, occlusal adjustments in the natural dentition and orthodontic treatment ${ }^{22,23}$, whereas a BBO follows the philosophy applied to removable complete dentures, seeking a better distribution of stresses and a consequent balance of the prosthesis ${ }^{3,10,13}$.

Consequently, it was hypothesized in this that the disocclusion in CGO would generate a more suitable stress distribution than the BBO in a modified Brånemark protocol prosthesis. This study compared the distribution of stresses generated by disocclusion in CGO and BBO in the implants and metallic infrastructure of a modified Brånemark protocol prosthesis without bilateral free distal ends.

\section{MATERIAL AND METHODS}

The SolidWorks program was used as a graphics tool to modify an existing geometric model developed by Greco, et al. ${ }^{7}$ (2009) and to insert the small implants. The new model was exported to the finite element program Abaqus ${ }^{\circledR} 2008$ (CAE Version 6.7, Providence, RI, USA) for the simulations. In this program, the whole model was meshed with tetrahedral elements. The previous mandible base model $^{6}$ was also developed using Solid Works ${ }^{\circledR}$ and then edited with Abaqus CAE in order to include the proposed system geometry and denture support.

Each component of the model had its mesh set separately and subsequently joined to obtain the complete model. The junction between each component pair (jaw-implants, implants-metallic infrastructure and metallic infrastructure-artificial teeth) was generated using the TIE command in the Abaqus commercial finite element program. As a consequence, each node in the contact surface of one component was constrained to move together with the adjacent node of the other component. This model contained 148,399 elements and 33,964 nodes. The mesh was tested and refined in the areas of interest until the response did not change significantly.

The new model simulated a mandible with seven titanium implants as pillars, five of them installed between the mental foramen, with a distance of $4 \mathrm{~mm}$ between their platforms. All of these types of implants were cylindrical, $13 \mathrm{~mm}$ in height and $3.75 \mathrm{~mm}$ in diameter (Brånemark System ${ }^{\circledR} \mathrm{Mk}$ III Groovy; Nobel ${ }^{\circledR}$ Biocare, Zürich-Flughafen, Switzerland). The drawings of the two posterior implants, located at the midpoint of the occlusal surface of the first molar, were cylindrical, $5 \mathrm{~mm}$ in height and $5 \mathrm{~mm}$ in diameter (Titamax WS ${ }^{\circledR}$; Neodent ${ }^{\circledR}$, Curitiba, PR, Brazil). The simulated prosthetic components made of titanium were $3 \mathrm{~mm}$ in height (Multi-unit Abutment ${ }^{\circledR}$; Nobel Biocare ${ }^{\circledR}$ ), providing a distance of $3 \mathrm{~mm}$ between the base of the infrastructure of the prosthesis and the bone surface (Figure 1).

A complete denture supported by implants was designed with a nickel-chromium infrastructure (Wiron ${ }^{\circledR}$ BEGO, Goldschlangerar, Bremen, Germany), a thickness of $6 \mathrm{~mm}$, a height of $4 \mathrm{~mm}$ and a total length of $112 \mathrm{~mm}$ with free distal ends. On this structure, the twelve elements in artificial dental acrylic resin (the first mandibular left first molar to the lower right) and a range of $2 \mathrm{~mm}$ gingival from the resin and without mucosal contact tissue were designed (Figure 1).

Implants 1 and 7 with lengths of $5 \mathrm{~mm}$ were considered the short implants near to the first molar on the working and balancing sides, respectively. Implants 2 and 6 with lengths of $13 \mathrm{~mm}$ were 
situated close to the canine area on the working and balancing sides, respectively. Implants 3, 4 and 5 were situated at the mental area between implants 2 and 6.

The Poisson's ratio and elasticity modulus of

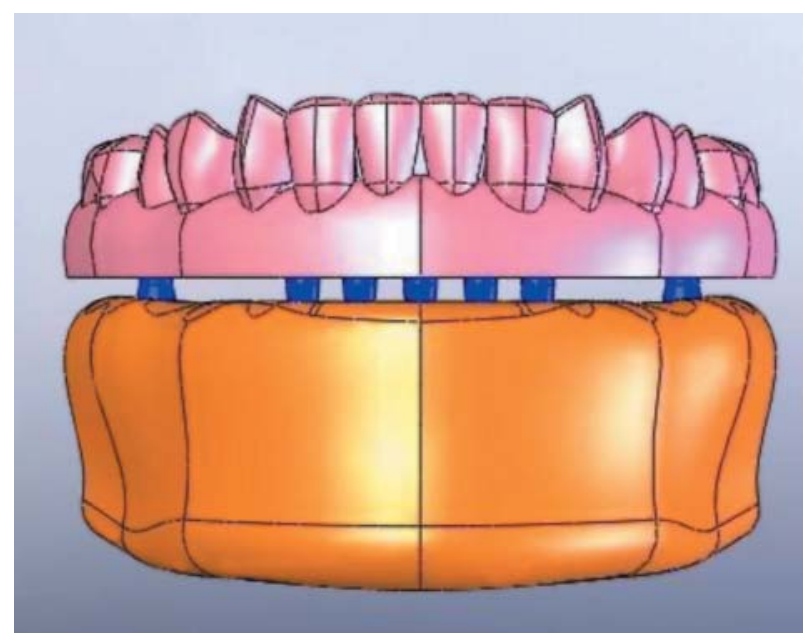

Figure 1- Model of the three-dimensional finite elements
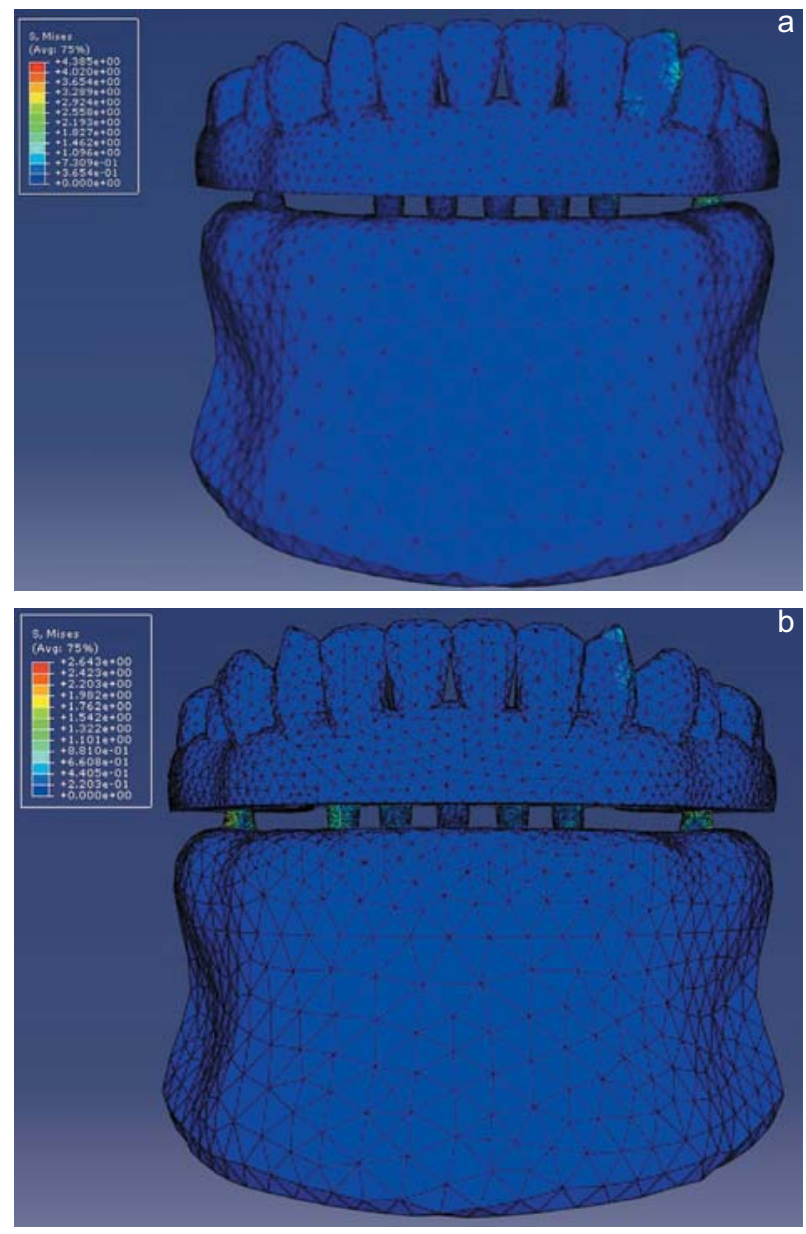

Figure 2- Distribution of the equivalent stress generated by disocclusion in canine guide occlusion (CGO) (a) and bilateral balanced occlusion (BBO) (b) on the complete model different materials that make up the models were determined according to the literature ${ }^{5,7,8,18}$ (Table 1).

Patterns of disocclusion were simulated by applying a nodal load of $15 \mathrm{~N}$ with an angle of $45^{\circ}$ to the canine tooth near implant 2 . On the standard of the canine guide, the point of contact was the vestibular incisal region of the canine on the working side. In the bilateral balanced occlusion, the points were distributed among the canine on the working side in the same region as that in the simulation of the CGO, the external part of the buccal mesial and distal vestibule of the first molar on the working side and the internal aspects of the mesial buccal atrium and distal first molar on the balancing side.

A load of $15 \mathrm{~N}$ in the simulation of the CGO was applied to one tooth, and the load in the simulation of the BBO was distributed across three teeth. Because the stress distribution in the teeth was not relevant for the analysis, no special precaution was taken regarding the local stress concentration at the point of load application.

The results were based on a linear elastic static analysis and were used to compare the magnitude of the equivalent stress for each of the simulations.

\section{RESULTS}

The stress distributions generated by disocclusion in $\mathrm{CGO}$ and $\mathrm{BBO}$ on the complete model are shown in Figures $2 \mathrm{a}$ and $2 \mathrm{~b}$. In $\mathrm{CGO}$, the equivalent stresses were concentrated at implants 1 and 2 on the working side. In $\mathrm{BBO}$, the equivalent stresses were distributed among implants 1, 6 and 7 .

The stress distributions generated by disocclusion in CGO and BBO on the implants and metallic infrastructure are shown in Figures $3 a$ and $3 b$. In the CGO, the equivalent stresses were concentrated in decreasing order at the neck of implant 1 , on the infrastructure on the working side and at the fixing screw of implant 4 . In $\mathrm{BBO}$, the equivalent stresses were concentrated in decreasing order at implants 1 and 7 , at the neck of implant 6 , on the infrastructure on the working side and balancing side and at the fixing screw at implant 4.

The stress distributions generated by disocclusion in CGO and BBO on the implants and metallic infrastructure are shown in Figures $3 \mathrm{a}$ and $3 \mathrm{~b}$. In CGO, the equivalent stresses were concentrated at the infrastructure on the working side. In the BBO, the equivalent stresses were distributed among the infrastructure on the working and balancing sides.

Figure 5 illustrates the equivalent stress distribution in each of the seven implants. In CGO, the peak of maximum stress occurred at implant 1 , followed by intermediate values at implant 2 and lower stress at the others implants. In BBO, implants 1 and 7 received the maximum stress 
values. Implants 1 and 2 received approximately two-fold more stress in the CGO than in the BBO. Implants 1 and 7 received similar stresses in
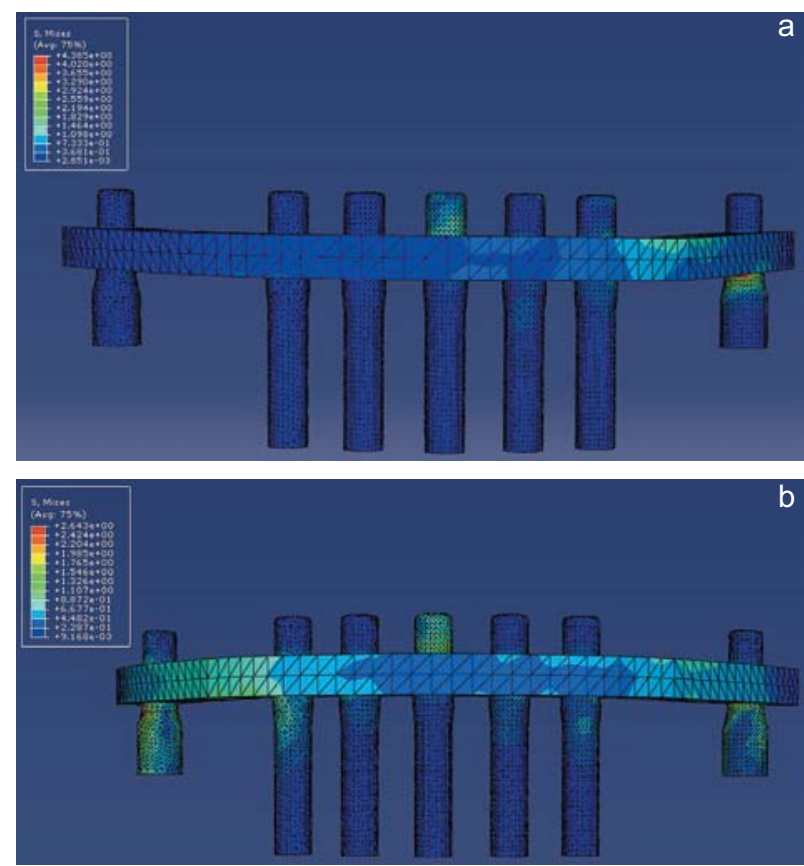

Figure 3- Distribution of the equivalent stress generated by disocclusion in canine guide occlusion (CGO) (a) and bilateral balanced occlusion (BBO) (b) on the implants and metallic infrastructure. Frontal view
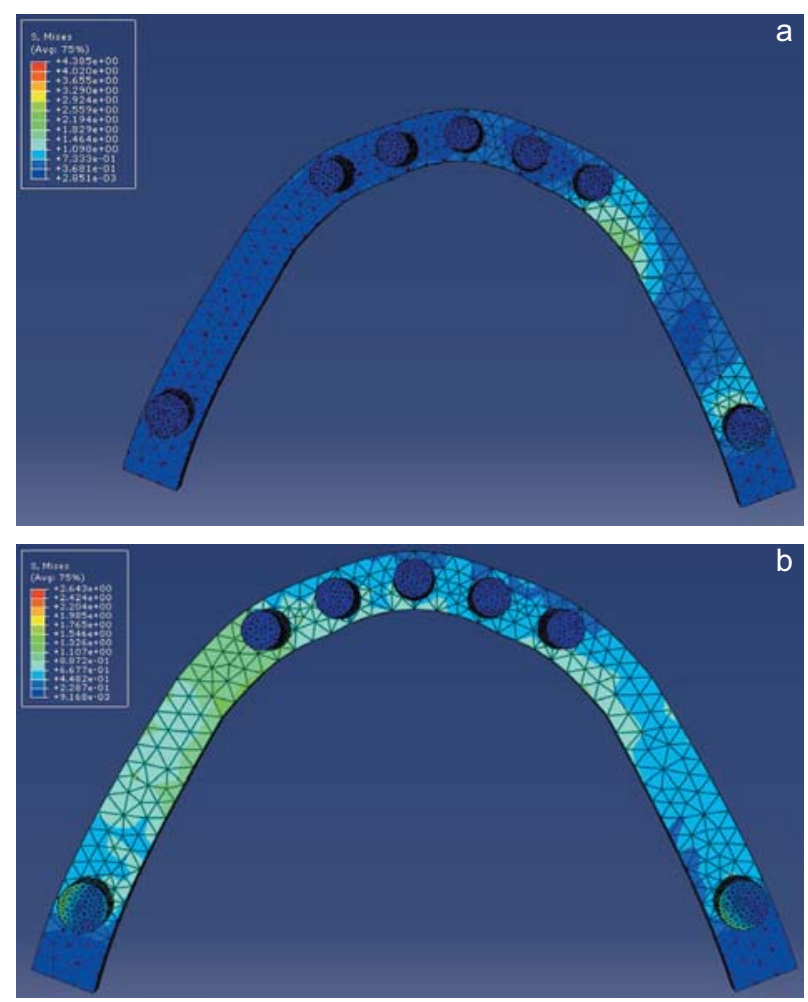

Figure 4- Distribution of the equivalent stress generated by disocclusion in canine guide occlusion (CGO) (a) and bilateral balanced occlusion (BBO) (b) on the implants and metallic infrastructure. Bottom view

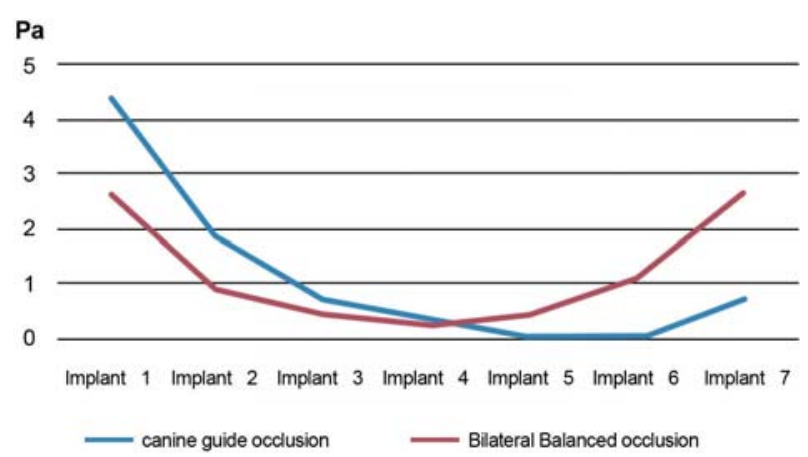

Figure 5- Values of the equivalent stress found in the implants

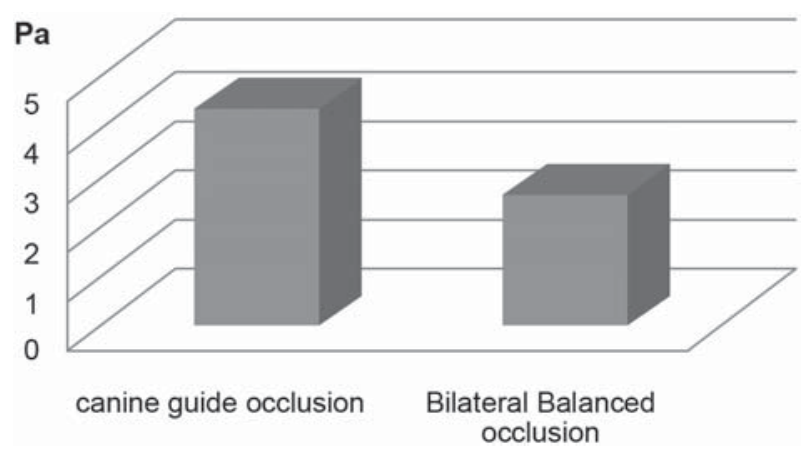

Figure 6- Values of the maximum stress in the patterns of disocclusion

the BBO as shown by a regular curve of stress distribution.

The value of the maximum stress found in the simulation of the pattern of disocclusion in CGO was two times greater than that in the simulation of BBO. This point of maximum stress in the two models was found at the neck of the short implant on the working side, located in the region of the first molar (Figure 6).

\section{DISCUSSION}

Several authors support the use of the BBO standard in complete denture conventional rehabilitation $3,6,17,23,29$, whereas unclamping in CGO is devoted to rehabilitation supported by teeth $10,13,22,26,27$.

The implant-supported prosthesis is the union of the concepts of the conventional complete denture and the fixed prosthesis supported by teeth. Originally, the pattern of chosen for disocclusion was $\mathrm{BBO}^{2}$. In a previous study using FEM, it was observed that the complete denture supported by implants with bilateral free ends of the Brånemark protocol type should be designed to receive the pattern of disocclusion in CGO, for which stresses were three times smaller than in BBO. For this type of prosthesis, the concentration of contact in the region of the canines was more favorable because it did not generate contact in the extreme areas of 
the free ends ${ }^{8}$.

It is observed, both qualitatively and quantitatively, that the literature still has poor evaluations of the effects of stresses generated on bone structures. The modeling of these structures by image processing and FEM biomechanical analyses is an alternative to address this issue. These approaches have the advantage of not being invasive, therefore contributing to studies such as the calculation of stress, strain and displacement at the bone-implant interface that would be impractical without them ${ }^{5,7}$. However, the FEM analysis of the distribution and absorption of generated tensions by the standards of occlusion and disocclusion in a prosthesis supported by implants should be treated with caution. The methodological limitations of a virtual simulation of the stress distribution in a prosthetic system must be considered $4,5,12,15,30$. Some methodological aspects should be highlighted. The load (15 N) was determined by convenience not to compromise the qualitative analysis of the stress distribution. If the load of disocclusion applied was $150 \mathrm{~N}$, the distribution of qualitative strains would be similar, whereas the quantitative analysis would show proportionally larger values. Other authors have been concerned with the evaluation of the occlusion, limiting the analysis of stresses at the distal free ends ${ }^{11,19,21}$. Most of these studies carry loads directly onto the free end in spite of the coating material over the metallic infrastructure ${ }^{1,11,19,21,28}$. The analysis of the stress distribution in this new model of implants showed a higher stress concentration in the CGO simulation than in the BBO. This result can be explained because the BBO contact points are distributed across three points, whereas in the $\mathrm{CGO}$, the contact is concentrated at the canine on the working side.

When we analyzed the maximum stresses found on each of the implants, we noticed that the curve of the stress distribution in the BBO was concave, with the ends of the prosthesis suffering more stress than intermediate implants. Moreover, the curve with the pattern of disocclusion in the CGO generated a stress peak in the implants on the working side and a steep drop towards the intermediate implants and those on the balancing side.

This difference in stress behavior between the two patterns of disocclusion can be better measured by considering that the working and balancing sides take turns as the individual switches the movement side. This means that, in any contact movement that the individual performs with the $\mathrm{BBO}$, the intermediate implants will be preserved, while the implants located in regions of two canine strain quadrants will receive light stresses, and the short implants located in the posterior regions will receive slightly higher stresses than the others. However, none of the implants in any kind of contact movements receive a high peak of stress.

In the CGO, the implants on the working side receive greater stresses than the intermediate implants and the implants on the balancing side. The short implant on the working side showed the highest level of stress. When the stresses are alternated between the working and balancing sides, there will be a wide variation among the magnitudes of stress on the short implants, with a peak of very high stress at some time and very low stress at other times. This pattern of stress distribution with maximum peaks at the short implants on the working side could compromise the longevity of these implants.

If the stresses on the intermediate implants are lower than those within the areas of the canine and both distal short implants in the CGO and the unclamping in the BBO, perhaps the three intermediate implants could be removed to combine this model with the concepts of the "on all four" system. This system offers a simplification in the design of clinical cases of complete dentures supported by implants, with the help of prototyping and guide surgery, which allows the use of only four implants as pillars of the mandible and maxilla prosthesis. These implants have a distal tip in order to minimize the extension of the distal free ends ${ }^{14,20,25}$. Reducing the number from seven to four implants would be a new possibility for anchoring the complete denture with biological and economic advantages if confirmed by in vitro and in vivo studies.

The mechanism by which stresses are absorbed and distributed by the implants and the supporting structures is essential to the longevity of the prosthesis. The dimension of the implants affects the absorption system of stresses. It seems that the use of wider rather than longer implants is more important because stresses are located at the neck of the implant. The larger the diameter of the implants, the lower the stresses in the region of the implant neck, whereas an increase in the implant length does not interfere with the significant reduction of the resulting stresses ${ }^{10}$. In this study, the maximum stresses found in the two simulations were located in the neck of the short implant on the working side. Despite its larger diameter ( 5 $\mathrm{mm}$ ), this is an implant of only $5 \mathrm{~mm}$ in height, which can be a critical factor to the longevity of oral rehabilitation. As shown in Figure 4, this implant suffers high stresses on almost its entire surface. The previous implants of $13 \mathrm{~mm}$ in height receive tensions located on their coronal portion, whereas their apical part suffers much smaller stresses or are hardly affected.

The comparison between the results of this study 
and those of $\mathrm{Greco}^{8}$ (2009) shows that the stresses generated in the implant region of the canine on the working side are larger in the simulation of the prosthesis with the free ends than on the prosthesis with no free ends for either BBO or CGO. When using the distal implants, modifying the Brånemark protocol, the stress distribution is lower across the entire infrastructure and in all the implants, which would justify their indication.

The results of the present study rejected the hypothesis that the disocclusion in CGO generates a stress distribution more suitable than the bilateral balanced occlusion with a modified Brånemark protocol prosthesis. The standard of choice for the modified Brånemark protocol should be $\mathrm{BBO}$ because it generates less stress on the implant abutments. Further studies, especially in vivo investigations, are required of this new proposed protocol before it can be safely included in the arsenal of prosthetic rehabilitation.

\section{CONCLUSION}

According to the criteria and limitations established in this study, it is possible to conclude that the disocclusion in BBO induced lower tensions than those in CGO in a mandibular complete denture supported by implants without free distal ends (the modified Brånemark protocol).

\section{REFERENCES}

1- Assif D, Marshak B, Horowitz A. Analysis of load transfer and stress distribution by an implant-supported fixed partial denture. J Prosthet Dent. 1996;75(3):285-91.

2- Brånemark PI. Osseointegration and its experimental background. J Prosthet Dent. 1983;50(3):399-410.

3- Christensen C. The problem of the bite. Dent Cosmos. 1905;47(10): 1184-95.

4- Eskitascioglu G, Usumez A, Sevimay M, Soykan E, Unsal $E$. The influence of occlusal loading location on stresses transferred to implant-supported prostheses and supporting bone: a three-dimensional finite element study. J Prosthet Dent. 2004;91(2):144-50.

5- Gomes de Oliveira S, Seraidarian PI, Landre J Jr, Oliveira DD, Cavalcanti BN. Tooth displacement due to occlusal contacts: a three dimensional finite elements study. J Oral Rehabil. 2006;33(12):874-80

6- Granger ER. Functional relations of the same stomatognathic system. J Am Dent Assoc. 1954;4(6):638-47.

7- Greco GD, Jansen WC, Landre Junior J, Seraidarian PI. Analysis of the functional stresses in the mandibular complete denture supported by implants, generated for distinct standards of occlusion. Braz Oral Res. 2009;23(2):175-81.

8- Greco GD, Jansen WC, Landre Junior J, Seraidarian PI. Biomechanical analysis of the stresses generated by different patterns disocclusion in an implant-supported mandibular complete denture. J Appl Oral Sci. 2009;17(5):515-20.
9- Guichet DL, Yoshinobu D, Caputo AA. Effect of splinting and interproximal contact tightness on load transfer by implant restorations. J Prosthet Dent. 2002;87(5):528-35.

10- Heartwell CM, Rahn AO. Syllabus of complete dentures. $2^{\text {nd }}$ ed. Philadelphia: Lea \& Febiger; 1974. p. 197-206.

11- Himmlová L, Dstálová T, Kácovský A, Konvicková S. Influence of implant length and diameter on stress distribution: a finite element analysis. J Prosthet Dent. 2004;91(1):20-5.

12- Huang HL, Fuh LJ, Hsu JT, Tu MG, Shen YW, Wu CL. Effects of implant surface roughness and stiffness of grafted bone on an immediately loaded maxillary implant: a 3D numerical analysis. J Oral Rehabil. 2008;35(4):283-90.

13- Jankelson B, Hoffman GM, Hendron JA Jr. The physiology of the stomatognathic system. J Am Dent Assoc. 1953;46(4):375-86. 14- Jensen OT, Adams MW. The maxillary M-4: a technical and biomechanical note for all-on-4 management of severe maxillary atrophy - report of 3 cases. J Oral Maxillofac Surg. 2009;67:173944.

15- Korioth TWP, Johann AR. Influence of mandibular superstructure shape on implant stresses during simulated posterior biting. J Prosthet Dent. 1999;82(1):67-72.

16- Krämer A, Weber $H$, Benzing $U$. Implant and prosthetic treatment of the edentulous maxilla using a bar-supported prosthesis. J Oral Maxillofac Implants. 1992;7(2):251-5.

17- Landa JS. Biologic significance of balanced occlusion and balanced articulation in complete denture service. J Am Dent Assoc. 1962;65(4):489-94.

18- Lin CL, Wang JC, Kuo YC. Numerical simulation on the biomechanical interactions of tooth/implant-supported system under various occlusal forces with rigid/non-rigid connections. J Biomech. 2006;39(3):453-63.

19- Lundgren D, Laurell L. Biomechanical aspects of fixed bridgework supported by natural teeth and endosseous implants. Periodontol 2000. 1994;4(1):23-40.

20- Malo P, Araujo Nobre M, Lopes A. The use of computer-guided flapless implant surgery and four implants placed in immediate function to support a fixed denture: preliminary results after a mean follow-up period of thirteen months. J Prosthet Dent. 2007;97(6):S26-S34.

21- Misch CE, Bidez MW. Implant-protected occlusion: a biomechanical rationale. Compendium. 1994;15(11):1330-4.

22- Nagao M. Comparative studies on the curve of Spee in mammals, with a discussion of its relation to the form of the fossa mandibular. J Dent Res. 1919;1(2):159-202.

23- Nairn RI. Lateral and protrusive occlusions. J Dent. 1973;1(4):181-7.

24- Ogawa T, Ogimoto T, Koyano K. Validity of the examination method of occlusal contact pattern relating to mandibular position. J Dent. $2000 ; 28(1): 23-9$.

25- Sanna AM, Molly L, Van Steenberghe D. Immediately loaded CAD-CAM manufactured fixed complete dentures using flapless implant placement procedures: a cohort study of consecutive patients. J Prosthet Dent. 2007;97(6):331-9.

26- Shaw DM. Form and function in the teeth. Int J Orthodont. 1924;10(11):703-18.

27- Sheppard IM. Denture base dislodgment during mastication. J Prosthet Dent. 1963;13(3):462-8.

28- White SN, Caputo AA, Anderkvist T. Effect of cantilever length on stress transfer by implant-supported prostheses. J Prosthet Dent. 1994;71(5):493-9.

29- Woda A, Vigneron P, Kay D. Nonfunctional and functional occlusal contacts: a review of the literature. J Prosthet Dent. 1979;42(3):35-41.

30- Zampelis A, Rangert B, Heijl L. Tilting of splinted implants for improved prosthodontic support: a two dimensional finite element analysis. J Prosthet Dent. 2007;97(6):S35-S43. 